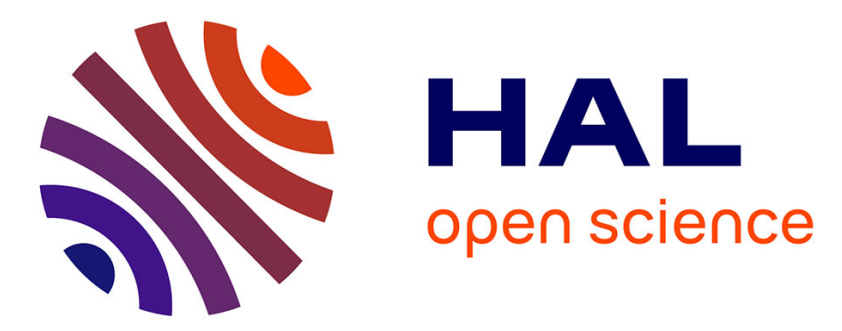

\title{
Deep-level transient spectroscopy of interfacial states in "buffer-free" p-i-n GaSb/GaAs devices
}

\author{
Mohsin Aziz, Philippe Ferrandis, Abdelmadjid Mesli, Riaz Hussain Mari, \\ Jorlandio Francisco Felix, Azzouz Sellai, Dler Jameel, Noor Al Saqri, \\ Almontaser Khatab, David Taylor, et al.
}

\section{To cite this version:}

Mohsin Aziz, Philippe Ferrandis, Abdelmadjid Mesli, Riaz Hussain Mari, Jorlandio Francisco Felix, et al.. Deep-level transient spectroscopy of interfacial states in "buffer-free" p-i-n GaSb/GaAs devices. Journal of Applied Physics, 2013, 114 (13), pp.134507. 10.1063/1.4824378 . hal-01616521v2

\section{HAL Id: hal-01616521 \\ https://hal.science/hal-01616521v2}

Submitted on 26 Jan 2018

HAL is a multi-disciplinary open access archive for the deposit and dissemination of scientific research documents, whether they are published or not. The documents may come from teaching and research institutions in France or abroad, or from public or private research centers.
L'archive ouverte pluridisciplinaire HAL, est destinée au dépôt et à la diffusion de documents scientifiques de niveau recherche, publiés ou non, émanant des établissements d'enseignement et de recherche français ou étrangers, des laboratoires publics ou privés. 


\section{Deep-level transient spectroscopy of interfacial states in "buffer-free” p-i-n GaSb/GaAs devices}

Mohsin Aziz, Philippe Ferrandis, Abdelmadjid Mesli, Riaz Hussain Mari, Jorlandio Francisco Felix, Azzouz Sellai, Dler Jameel, Noor Al Saqri, Almontaser Khatab, David Taylor, and Mohamed Henini

Citation: Journal of Applied Physics 114, 134507 (2013);

View online: https://doi.org/10.1063/1.4824378

View Table of Contents: http://aip.scitation.org/toc/jap/114/13

Published by the American Institute of Physics

\section{Articles you may be interested in}

Deep-level transient spectroscopy: A new method to characterize traps in semiconductors

Journal of Applied Physics 45, 3023 (2003); 10.1063/1.1663719

Strain relief by periodic misfit arrays for low defect density GaSb on GaAs

Applied Physics Letters 88, 131911 (2006); 10.1063/1.2172742

Formation of periodic interfacial misfit dislocation array at the InSb/GaAs interface via surface anion exchange Journal of Applied Physics 120, 035301 (2016); 10.1063/1.4958863

Constant-capacitance DLTS measurement of defect-density profiles in semiconductors Journal of Applied Physics 50, 4828 (2008); 10.1063/1.326546

Electronic characteristics of the interfacial states embedded in "buffer-free" GaSb/GaAs (001) heterojunctions Applied Physics Letters 95, 202107 (2009); 10.1063/1.3266835

Laplace-transform deep-level spectroscopy: The technique and its applications to the study of point defects in semiconductors

Journal of Applied Physics 96, 4689 (2004); 10.1063/1.1794897

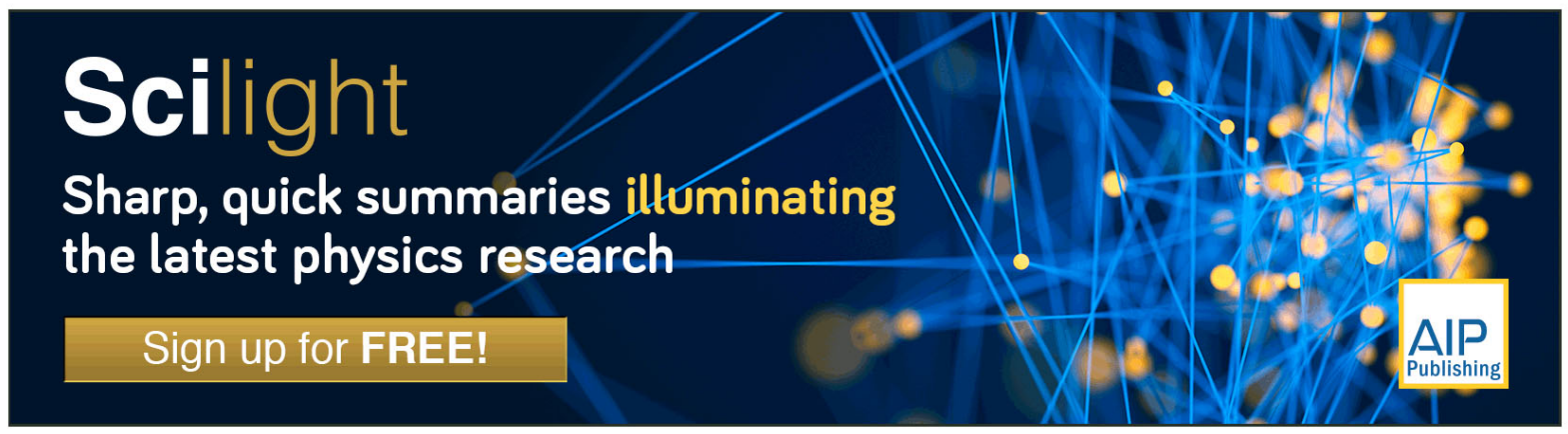




\title{
Deep-level transient spectroscopy of interfacial states in "buffer-free" p-i-n GaSb/GaAs devices
}

\author{
Mohsin Aziz, ${ }^{1}$ Philippe Ferrandis, ${ }^{2}$ Abdelmadjid Mesli, ${ }^{2}$ Riaz Hussain Mari, ${ }^{1}$ \\ Jorlandio Francisco Felix, ${ }^{3}$ Azzouz Sellai, ${ }^{4}$ Dler Jameel, ${ }^{1}$ Noor Al Saqri, ${ }^{1,4}$ \\ Almontaser Khatab, ${ }^{1}$ David Taylor, ${ }^{1}$ and Mohamed Henini ${ }^{1, a)}$ \\ ${ }^{1}$ School of Physics and Astronomy, Nottingham Nanotechnology and Nanoscience Center, \\ University of Nottingham, Nottingham NG7 2RD, United Kingdom \\ ${ }^{2} I M 2 N P, C N R S$ (UMR 7334), and Université Aix-Marseille, 13397 Marseille Cedex 20, France \\ ${ }^{3}$ Departamento de Física - Universidade Federal de Viçosa, 36570-000 Viçosa, Minas Gerais, Brazil \\ ${ }^{4}$ Department of Physics, Sultan Qaboos University, Oman
}

(Received 13 May 2013; accepted 20 September 2013; published online 2 October 2013)

\begin{abstract}
A systematic study was carried out on defect states in Interfacial Misfit (IMF) unpassivated and Tepassivated IMF in p-i-n GaSb/GaAs devices using Deep Level Transient Spectroscopy (DLTS) and Laplace DLTS. Additionally, Current-Voltage $(I-V)$ measurements were performed, which showed that the turn-on voltage $\left(V_{o n}\right)$ of passivated samples is lower than that for unpassivated samples; an effect which can be explained by the introduction of new defects states near to the interface of $\mathrm{GaSb} / \mathrm{GaAs}$, where Te was incorporated to passivate the IMF. The Capacitance-Voltage $(C-V)$ analysis demonstrates that these new states are the consequence of adding Te at the misfit of $\mathrm{GaSb} / \mathrm{GaAs}$. Furthermore, DLTS measurements reveal a distribution of states including a main midgap energy level, namely the well documented EL2 trap, with some peculiar behaviour. Most of these levels are related to interface states that are generated by the mismatch between GaAs and GaSb. Originally, the addition of Te atoms was thought to passivate these interface states. On the contrary, this paper, which attempts at correlating the current-voltage and capacitance-voltage characteristics to the DLTS results, shows clearly that Te atoms increase the density of interface states. (C) 2013 AIP Publishing LLC. [http://dx.doi.org/10.1063/1.4824378]
\end{abstract}

\section{INTRODUCTION}

$\mathrm{GaSb}$ is a direct band gap material with an energy gap of $0.72 \mathrm{eV}$. Due to its narrow band gap, it has potential applications in long wavelength optoelectronic devices; ${ }^{1,2}$ solar cells $^{3}$ and quantum dots (QDs) memory devices. ${ }^{4}$ The research on GaSb material system has increased considerably, ${ }^{5}$ due to its advantage to grow it on low-cost GaAs substrates as compared to the native substrate.

By using Interfacial Misfit (IMF) growth mode, the strain between the substrate and epitaxial layer is, in principle, relieved by the formation of two-dimensional misfit array parallel to the interface between $\mathrm{GaAs}$ and $\mathrm{GaSb}$; these are referred to as $90^{\circ}$ dislocations. ${ }^{6-8}$ The strain relief in $90^{\circ}$ misfit is completely along [110] and [110] direction. The well-known threading dislocations in $\mathrm{GaAs} / \mathrm{GaSb}$ are caused by the $60^{\circ}$ misfit dislocations at the interface. These are formed to relieve the strain after the growth of a GaSb layer beyond the critical thickness. The undesirable threading dislocations can thread through the active region of a sample affecting the electrical and optical properties of the device. ${ }^{7}$ On the other hand, the $90^{\circ}$ dislocations, which are confined to the interface and propagate only laterally, are the efficient mechanism to relieve the strain. ${ }^{9}$ These $90^{\circ}$ IMF dislocations which create interfacial states are made of Ga-dangling bonds localized at the $\mathrm{GaSb} / \mathrm{GaAs}$ interface. These interfacial states significantly affect various electrical

\footnotetext{
${ }^{\text {a) }}$ Author to whom correspondence should be addressed. Electronic mail: mohamed.henini@nottingham.ac.uk
}

characteristics of devices, ${ }^{10}$ such as the turn-on voltage and the reverse-bias leakage current. ${ }^{11,12}$ It is therefore important to study their electronic properties. One of the major issues that affect the performance of the devices is the presence of Ga dangling bonds because they trap carriers. It is therefore essential to compensate these unwanted channels. The delta doping technique of the region near the interface between GaAs and $\mathrm{GaSb}^{12}$ by a suitable dopant atom has been used as an alternative approach to achieve this "compensation" as referred to in Refs. 11 and 12 (in the following, we will use the term passivation instead of compensation, which usually means that doping of opposite type is present in the sample, lowering the net doping. In addition, we will refer to our samples as unpassivated and passivated instead of uncompensated and compensated ${ }^{11,12}$ ). For example, it has been shown that the electronic properties of $\mathrm{GaSb} / \mathrm{GaAs}$ based devices can be improved by using $T e$ atoms. ${ }^{11,12}$ However, in order to understand the influence of the interfacial states on optoelectronic devices such as lasers, detectors, solar cells, it is vital to study the defects present near the $\mathrm{IMF}-\mathrm{GaSb} / \mathrm{GaAs}$ and their interactions with delta doping processes. In this work, electrically active defects present in IMF-GaSb/GaAs heterostructures were investigated for the first time by employing Deep Level Transient Spectroscopy (DLTS) technique.

\section{SAMPLE DETAILS}

The samples were grown using the same procedure as reported in Refs. 11 and 12. Briefly, the samples are grown 


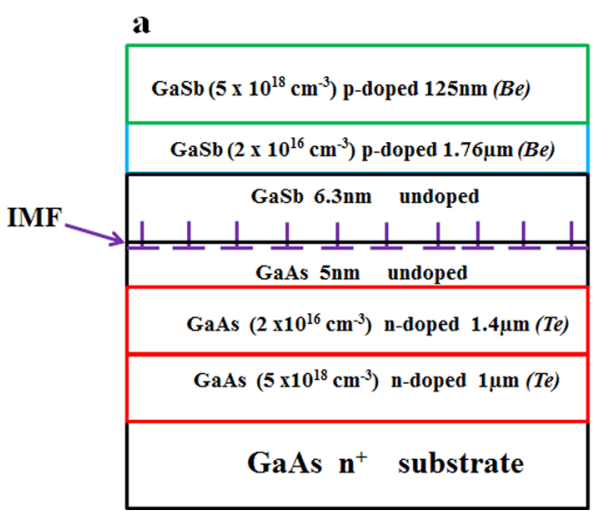

b

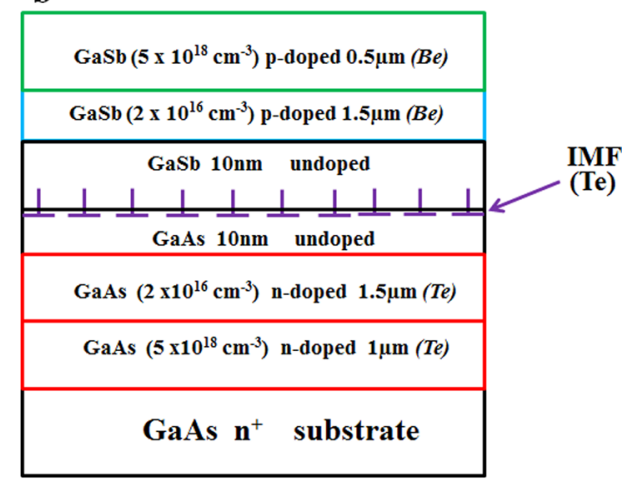

FIG. 1. Structure of (a) unpassivated (IMF before $\delta$-doping), and (b) passivated samples (after $\delta$-doping with Teatoms at IMF). by molecular beam epitaxy (MBE) on (100) GaAs substrates. After oxide removal, the RHEED pattern shows a $(2 \times 4)$ reconstruction demonstrating a high quality As rich stabilized GaAs surface. This was followed by desorption of As, which resulted in a $\mathrm{Ga}$ rich surface as confirmed by the $(4 \times 2)$ RHEED reconstruction. The IMF is then created by exposing the surface to $\mathrm{Sb}$ atoms. This results in a $(2 \times 8)$ reconstruction, which confirms the atomic packing instead of tetragonal distortion. The substrate temperature was then reduced and GaSb growth started resulting in a bulk $(1 \times 3)$ reconstructed GaSb growth. The layer structure of the devices investigated is shown in Figure 1.

In these structures, $B e$ and $T e$ are used as $p$ and $n$ type dopants, respectively. The doping concentration of the contact layers, i.e., $p$ - $G a S b$ and $n-G a A s$, for both unpassivated and passivated samples, are $5 \times 10^{18} \mathrm{~cm}^{-3}$, whereas for the other layers the doping is $2 \times 10^{16} \mathrm{~cm}^{-3}$. In unpassivated diodes, the $p-n$ junction is formed by a $1.76 \mu \mathrm{m}$ thick $\mathrm{GaSb}$ and $1.4 \mu \mathrm{m}$ GaAs layers on either side of the junction, respectively. The passivated $\mathrm{GaSb} / \mathrm{GaAs}$ devices with a sheet of $T e$ atoms at the interface will allow us to investigate and analyse the compensation of the interfacial states. In $\delta$-doped IMF diodes, the IMF region is embedded in a $10 \mathrm{~nm}$ unintentionally doped $\mathrm{GaSb}$ and $\mathrm{GaAs}$ regions on the respective sides of the interface. In unpassivated diodes, the IMF region is embedded between $6.3 \mathrm{~nm} \mathrm{GaSb}$ and $5 \mathrm{~nm}$ GaAs layers. The segregation of $\mathrm{Be}$ and $\mathrm{Te}$ dopants into the IMF region can be neglected since the samples were grown at relatively low temperatures. In addition, the undoped spacer layers on either side of the IMF region will further limit the diffusion of dopants into the IMF. The samples were processed into circular mesas having $200 \mu \mathrm{m}$ diameters.

\section{EXPERIMENTAL RESULTS}

In order to study the electrical properties of these IMF and to understand the role of compensation, $I-V, C-V$, and DLTS measurements were performed. Figure 2 represents the $I-V$ characteristics for both samples at room temperature.

The inset of Figure 2 shows the linear $I-V$ plot, where it can be seen that the turn-on voltage $\left(\mathrm{V}_{\text {on }}\right)$ of both samples is different. Jallipalli et al. ${ }^{11,12}$ explained the low value of $\mathrm{V}_{\text {on }}$ in their passivated samples as due to the compensation of interfacial states by Te atoms. It is worth pointing out the value of the turn-on voltage can only be considered as qualitative since there are several parasitic current paths, which shunt the hetero-barrier and make the I-V characteristics more complicated. Among the most important sources of currents, one may cite thermal generation of carrier in the semiconductor bulk or via deep level at the heterointerface, tunnelling across the narrow heterojunction barrier, and shunt current along the edge of the sample. All these sources will contribute to different extents to the measured current, thereby making it difficult to ascertain the value of the barrier limited current. However, the main contribution of the current is through the interfaces states resulting from the significant lattice mismatch between $\mathrm{GaSb}$ and $\mathrm{GaAs}$ in this material system. Therefore, the general trend is that the passivated samples exhibit a lower turn-on voltage, which is in agreement with the fact (and also as demonstrated throughout this manuscript) that the compensation process introduces more interface traps than it is supposed to suppress as claimed in the work reported by Jallipalli et al. ${ }^{11,12}$ These interface states increase dramatically the tunnelling current, and thereby reducing the turn-on voltage. In addition to the interface states, the current through the devices may be influenced by the series resistance effect as discussed by Chattopadhyay and Haldar. ${ }^{13}$ However, in the devices investigated in his work, the series resistance plays a minor role since the tunnelling current remained unchanged as demonstrated by recording the $\mathrm{I}-\mathrm{V}$ characteristics at different temperatures (data not shown here). One therefore can conclude

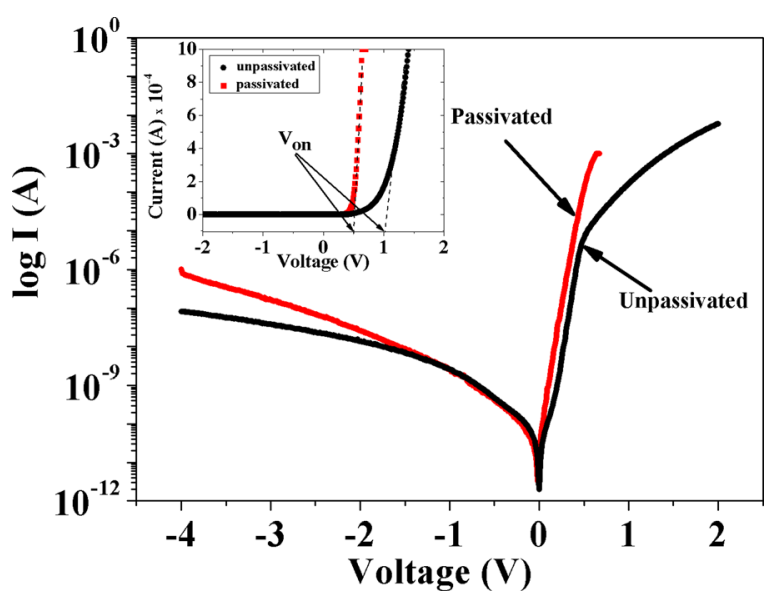

FIG. 2. Semi-logarithmic plots of dark I-V characteristics of unpassivated and passivated IMF diodes at room temperature with diameter of $200 \mu \mathrm{m}$. 
that the major contribution to the measured current in the passivated samples is due to the higher interface states.

However, by using room temperature $C-V$ measurements, it is found that the density of interfacial states in passivated samples is higher than in unpassivated samples. In this work by a careful analysis of the $C-V$ data, we explain that a lower value of $\mathrm{V}_{\text {on }}$ in the passivated samples is not necessary an evidence of lower interfacial states. The capacitance of an ideal heterojunction is given by ${ }^{14}$

$$
C=A\left(V_{\text {on }}+V_{R}\right)^{-1 / 2},
$$

where " $\mathrm{V}_{\text {on }}$ " is the diffusion or turn on voltage, " $V_{R}$ " is the applied reverse bias, and $A$ is a constant containing the doping concentration on both sides of the heterojunction (one for each material) and the corresponding dielectric constants. This proportionality factor can easily be derived in the case of an ideal heterojunction.

In any type of ideal, homo or heterojunction system plotting $1 / \mathrm{C}^{2}$ as a function of $\left(\mathrm{V}_{\text {on }}+\mathrm{V}_{\mathrm{R}}\right)$ or $\mathrm{C}$ as a function of $\left(\mathrm{V}_{\mathrm{on}}+\mathrm{V}_{\mathrm{R}}\right)^{-1 / 2}$ is obviously linear. However, there are cases where departure from linearity of either of the types of plotting may provide very instructive information. This aspect is fully exploited in our manuscript. The nonlinearity of $1 / C^{2}$ of heterojunctions, especially near $\left(V_{o n}+V_{R}\right)=0$, has been lengthily discussed in the literature. ${ }^{15}$ These nonlinearity effects are mainly caused by the interface states, which make the equivalent circuit much more complicated than a simple series connection of two equivalent devices (a capacitance in parallel with a conductance), representing each side of the heterojunction. In this work, we show that the simple parallel connection of two capacitances model as reported by Pandey and $\mathrm{Kal}^{16}$ one representing the total capacitance of the heterojunction (both sides included) and the other representing the interface contribution, fit our experimental data. In the following analysis, we will exploit the trivial but interesting case where the plot of $1 / \mathrm{C}^{2}$ as a function of $\left(\mathrm{V}_{\text {on }}\right.$ $\left.+V_{R}\right)$ is non-linear, while the plot of $C$ as a function of $\left(V_{\text {on }}\right.$ $\left.+\mathrm{V}_{\mathrm{R}}\right)^{-1 / 2}$ is linear, allowing the determination of valuable information of our structures.

As explained above, it is important to notice here that relying on Eq. (1), whether we plot $C$ versus $\left(V_{\text {on }}+V_{R}\right)^{-1 / 2}$ or $1 / C^{2}$ versus $\left(V_{\text {on }}+V_{R}\right)$, one should, in principle, obtain a linear behaviour for which the intercept is at the origin. This simple analysis has been applied to the data obtained at $300 \mathrm{~K}$ for both passivated and unpassivated samples. The two plots are shown in Figures 3 and $4 . V_{\text {on }}$ values are extracted from the $\mathrm{I}-\mathrm{V}$ characteristics at $300 \mathrm{~K}$.

It is absolutely clear from Figure 3 that $1 / C^{2}$ as a function of $\left(V_{o n}+V_{R}\right)$ is not linear, whereas plotting $\mathrm{C}$ as a function of $\left(\mathrm{V}_{\mathrm{on}}+\mathrm{V}_{\mathrm{R}}\right)^{-1 / 2}$ leads to a perfect straight line as shown in Figure 4 but with a nonzero intercept.

The $300 \mathrm{~K}$ plots shown in Figures 3 and 4 have similar behaviour for temperatures ranging from $20 \mathrm{~K}$ to $400 \mathrm{~K}$.

To account for the non-zero intercept in Figure 4, Eq. (1) must however be modified by adding a constant term to account for the non-zero intercept. This leads to

$$
C=A\left(V_{\text {on }}+V_{R}\right)^{-1 / 2}+C_{0} .
$$

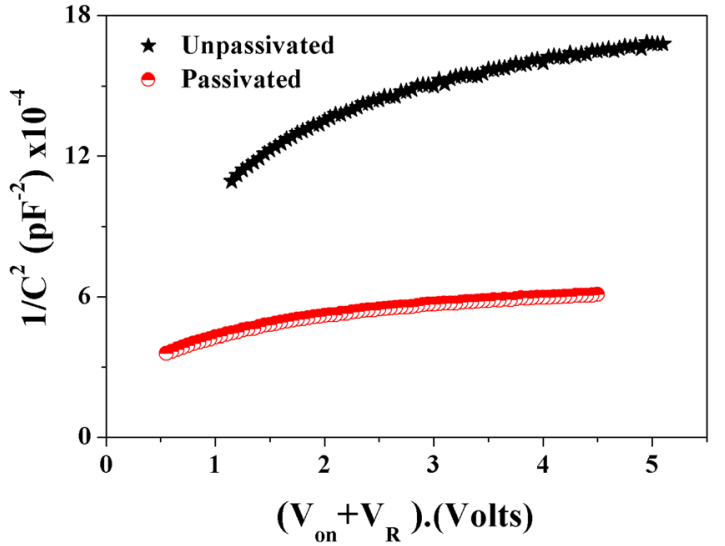

FIG. 3. Plot of $1 / C^{2}$ as a function of $\left(V_{o n}+V_{R}\right)$. The capacitance was measured at a frequency of $1 \mathrm{MHz}$. Clearly, there is no abrupt change of the charge distribution on both sides of the heterojunction.

The total capacitance of the device is thus equivalent to two separate capacitors in parallel; one due to the depletion region, called also the junction capacitance, and the second due to the interfaces states. It is worth pointing out that the term $\mathrm{C}_{0}$ is also affected by the value of $V_{o n}$ of the passivated and unpassivated samples as demonstrated in Figure 4. In order to explain the reason why the intercepts, i.e., values of $\mathrm{C}_{0}$, are different for both structures one needs to introduce some extra charges that add up to or subtract from the doping densities in the interface regions between $\mathrm{GaSb}$ and $\mathrm{GaAs}$ layers. Moreover, as extra charges are expected to affect the band bending, $V_{\text {on }}$ must also be affected. Due to the mismatch between the two materials, it is natural to assign the nonzero intercept observed in to the existence of a sheet layer ( $\delta$-layer) at the heterojunction that contains interface states with a total charge $\mathrm{Q}_{\mathrm{it}}$ and a density $\mathrm{D}_{\mathrm{it}}$. These two parameters are linked by the relation

$$
Q_{i t}= \pm q D_{i t}\left(E_{F s}-E_{0}\right),
$$

where the signs \pm refers to donors and acceptors, respectively, $\mathrm{E}_{\mathrm{Fs}}$ is the Fermi level at the interface and $\mathrm{E}_{0}$ is the

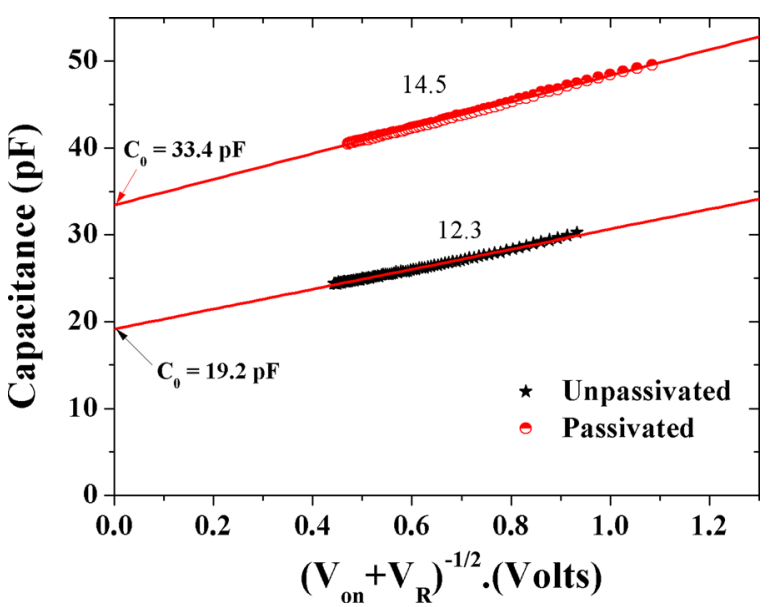

FIG. 4. Plot of $\mathrm{C}$ as a function of $\left(\mathrm{V}_{\mathrm{on}}+\mathrm{V}_{\mathrm{R}}\right)^{-1 / 2}$. The important result here is that the slope is slightly increased in the passivated samples. The corresponding $\mathrm{V}_{\text {on }}$ values of each device were extracted from the experimental data shown in Figure 2. 
so-called neutral level introduced originally by Bardeen ${ }^{17}$ to take into account the surface states at the interface between a metal and semiconductor. Avoiding detailed calculations, it is easy to show that in the ideal case, where there are no interface states, $\mathrm{C}_{0}$ should be equal to zero and $\mathrm{A}$ is given by

$$
A=\left[\frac{q \varepsilon_{0} N_{a} N_{d} \varepsilon_{1} \varepsilon_{2}}{2\left(N_{a} \varepsilon_{1}+N_{d} \varepsilon_{2}\right)}\right]^{1 / 2},
$$

where $\mathrm{N}_{\mathrm{a}}$ and $\mathrm{N}_{\mathrm{d}}$ are doping densities in $\mathrm{p}$ and $\mathrm{n}$ region of the diodes, and $\varepsilon_{1}$ and $\varepsilon_{2}$ are dielectric constants of GaSb and $\mathrm{GaAs}$, respectively. If one assumes that the incorporation of Te at the interface does not affect $\mathrm{N}_{\mathrm{a}}$ and $\mathrm{N}_{\mathrm{d}}$, or very negligibly, then Eq. (4) can be simplified since in our samples $\mathrm{N}_{\mathrm{a}}=\mathrm{N}_{\mathrm{d}}=2 \times 10^{16} \mathrm{~cm}^{-3}$. However, according to the $C-V$ results, it can be concluded that while compensating the IMF, Te-delta doping introduces extra charge states breaking down the symmetry of the doping in the passivated samples, i.e., $\mathrm{N}_{\mathrm{a}}$ is no longer equal to $\mathrm{N}_{\mathrm{d}}$. It would then be more reasonable to consider that the difference in slopes in both structures (12.3 in unpassivated and 14.5 in passivated) can be accounted for by the Te modification of $\mathrm{N}_{\mathrm{a}}$ and/or $\mathrm{N}_{\mathrm{d}}$.

Furthermore, in order to confirm the existence of interface states in both samples, capacitance (C) as function of frequency (f) measurements was performed. It well-known that the higher values of capacitance at low frequencies are attributed to the excess capacitance resulting from the interface states as reported by other workers. ${ }^{18,19}$ Figure 5 shows room temperature C-f characteristics for a DC-bias of zero Volts for both samples. It can clearly be seen from Figure 5 that the capacitance is significantly higher at low frequencies and drops considerably as the frequency increases. This is seen as an evidence of the presence of interface states in both samples. As long as these interface states are able to follow the variations of the AC test signal at low frequencies, they contribute to the measured capacitance. While at higher frequencies, the interface states are not able to follow the AC test signal (capture-emission rates are much slower) and as a result only the junction capacitance persists. It can also be noted from Figure 5 that $T e$ passivated samples have much higher capacitance than unpassivated devices, which further supports our above $\mathrm{CV}$ analysis.

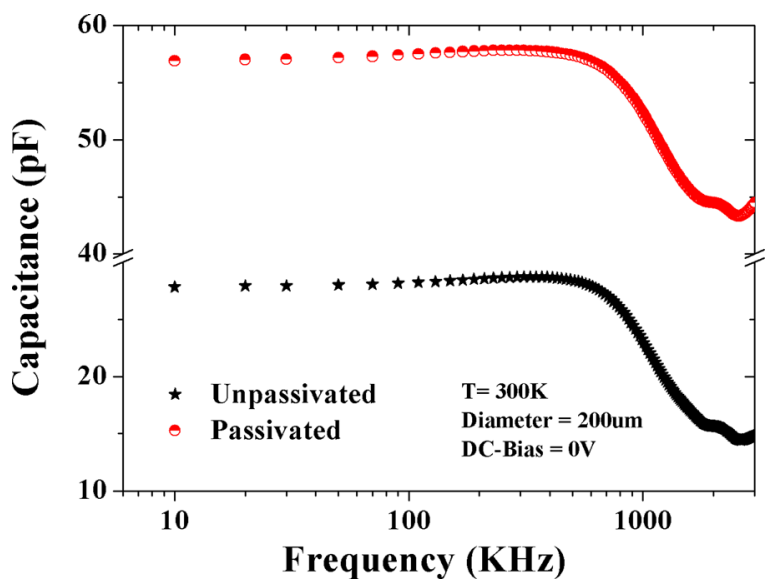

FIG. 5. Room temperature capacitance versus frequency (f) characteristics of unpassivated and passivated samples.
In order to understand the interaction of IMF and Te, and its influence on the electronic transport mechanism, it is important to study the electrically active defects in both samples. DLTS and Laplace DLTS are powerful techniques to detect traps in the bandgap of semiconductor materials. The details of these techniques are given elsewhere. ${ }^{20,21}$

DLTS experiments were performed at different reverse bias conditions. By applying a reverse bias, one can control the depletion region, where trapping and de-trapping of charge carriers processes occur. This will allow the measurement of the emissions of trapped carriers at a particular region of the device. The DLTS signals from the unpassivated samples, recorded at different reverse biases starting from $V_{R}=-0.25 \mathrm{~V}$ (very close to the interface) to $V_{R}=-4 \mathrm{~V}$ (away from the interface) are displayed in Figure 6. The filling pulse was kept constant at $\mathrm{V}_{\mathrm{p}}=0 \mathrm{~V}$ and $\mathrm{t}_{\mathrm{p}}=1 \mathrm{msec}$ duration for all the measurements.

It can be seen that for a reverse bias $\mathrm{V}_{\mathrm{R}}=-0.25 \mathrm{~V}$ (the region probed is near the interface) only one peak at about $383 \mathrm{~K}$ is detected. The isothermal Laplace DLTS technique in which the emission rate is measured at a constant temperature has been applied to get deeper insight into this well-defined peak. By plotting the temperature versus emission rate, one can obtain the energy of the trap. From the Arrhenius plot shown in Figure 7, activation energy of $0.79 \mathrm{eV}$ (defect $\mathrm{E}_{\mathrm{Up} 5}$ ) was determined with an apparent capture cross-section of $1.14 \times 10^{-12} \mathrm{~cm}^{2}$. The inset illustrates the Laplace DLTS peak. The full width at half maximum (FWHM) of the single peak detected by conventional DLTS at $\mathrm{V}_{\mathrm{R}}=-0.25 \mathrm{~V}$ (see Figure 6) is lower than $0.1 \mathrm{~T}_{\mathrm{m}}$ (where $\mathrm{T}_{\mathrm{m}}$ is the maximum peak intensity), indicating that a single level is present. ${ }^{22}$ This is clearly confirmed by the high resolution Laplace DLTS measurements in the inset of Figure 7.

Increasing the reverse bias from $\mathrm{V}_{\mathrm{R}}=-0.25 \mathrm{~V}$ to $\mathrm{V}_{\mathrm{R}}=-4 \mathrm{~V}$ leads to another increasing signal on the low temperature side of the main peak. This observation means that we are not necessarily probing a single state as will be demonstrated below. Above $\mathrm{V}_{\mathrm{R}}=-1 \mathrm{~V}$, another peak starts appearing at a temperature of $\sim 217 \mathrm{~K}$ (for clarity, this

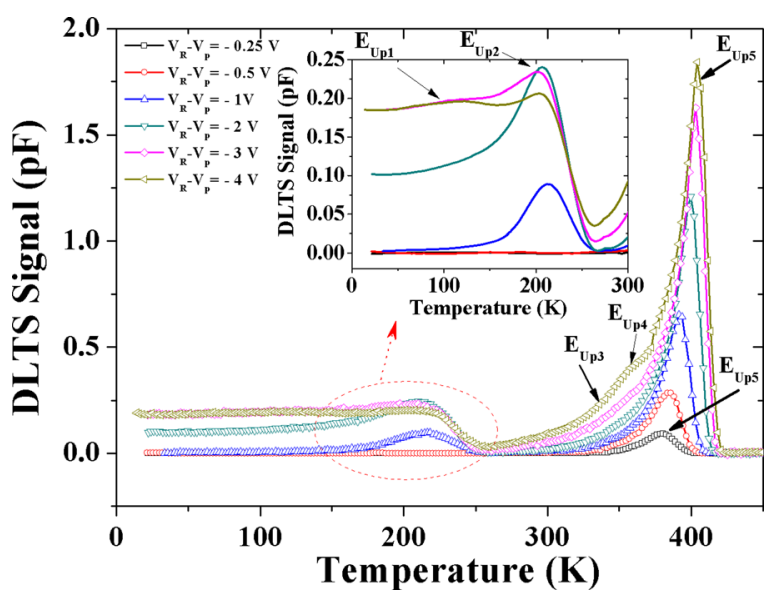

FIG. 6. Conventional DLTS measurements of unpassivated samples at different reverse biases from $V_{R}=-0.25 \mathrm{~V}$ to $V_{R}=-4 \mathrm{~V}$. The filling pulse characteristics were: $\mathrm{V}_{\mathrm{p}}=0 \mathrm{~V}, \mathrm{t}_{\mathrm{p}}=1 \mathrm{msec}$, and rate window $=200 \mathrm{~s}^{-1}$. For clarity, the low temperature peaks are shown in the inset. 


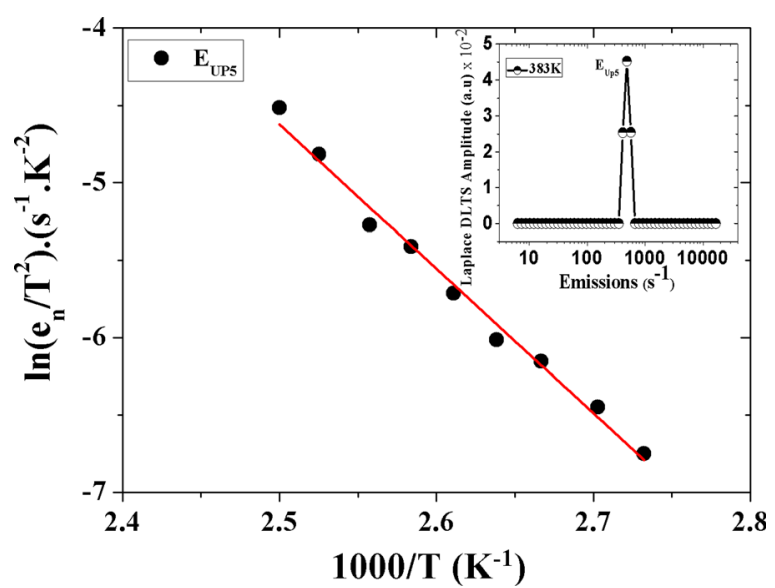

FIG. 7. Arrhenius plot of unpassivated samples at reverse bias $\mathrm{V}_{\mathrm{R}}=-0.25 \mathrm{~V}$. The inset shows Laplace DLTS peak at $383 \mathrm{~K}$.

additional DLTS peak is shown in the inset of Figure 6) and broadens on the left hand side (lower temperature) when the bias is increased further up to $\mathrm{V}_{\mathrm{R}}=-4 \mathrm{~V}$. This broadening is typical for tunnelling processes through the interface between the two materials, namely, GaAs and GaSb that have different band gaps. As the band bending increases with applied bias, the thickness of the triangular barrier reduces accordingly allowing the carriers trapped at or close to the interface to escape easily by tunnelling. ${ }^{23}$

On the other hand, the peak that was very well resolved at the low reverse bias of $-0.25 \mathrm{~V}$ becomes somehow distorted when the bias increases up to $-4 \mathrm{~V}$ suggesting that more than one level exist (Figure 6). To elucidate this further, the Laplace DLTS was done at reverse bias of $\mathrm{V}_{\mathrm{R}}=-4 \mathrm{~V}$ and it can be clearly seen from the inset of Figure 8 that there are three peaks $\left(\mathrm{E}_{\mathrm{Up} 3}, \mathrm{E}_{\mathrm{Up} 4}, \mathrm{E}_{\mathrm{Up} 5}\right)$, one of which $\left(\mathrm{E}_{\mathrm{Up} 5}\right)$ has exactly the same activation energy and capture cross-section as those derived at the reverse bias of $\mathrm{V}_{\mathrm{R}}=-0.25 \mathrm{~V}$ in the unpassivated samples. On the other hand, for the peak that appears at $217 \mathrm{~K}$ (large "plateau" at low temperatures for higher reverse bias values) in

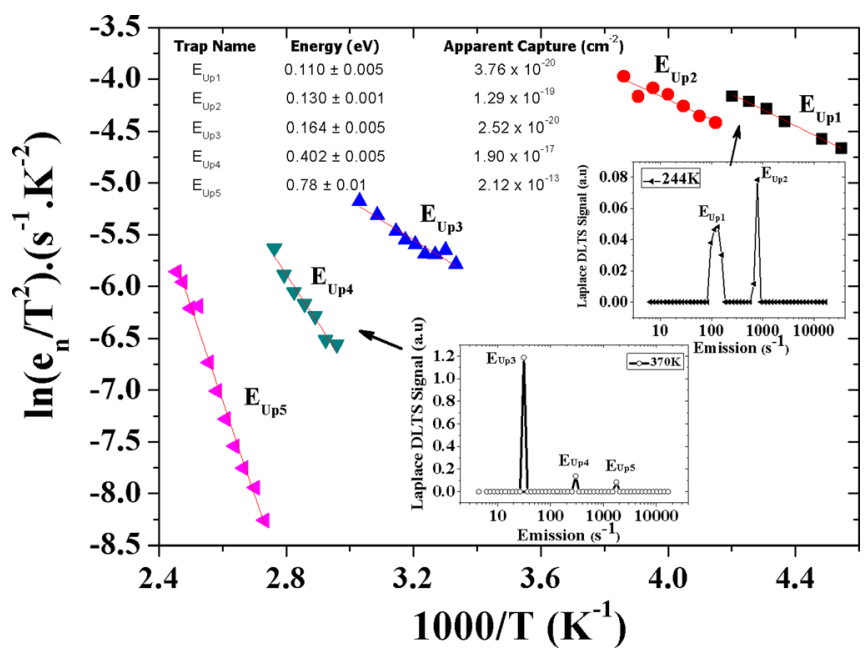

FIG. 8. Arrhenius plot of unpassivated samples at reverse bias $V_{R}=-4 \mathrm{~V}$. The red line represents the linear fit to the data. The inset shows that the main DLTS peak observed for $\mathrm{V}_{\mathrm{R}}=-0.25 \mathrm{~V}$ (as shown in Figure 6) splits in three clear peaks as detected by the high resolution Laplace DLTS. unpassivated samples, the Laplace DLTS reveals two closely spaced peaks $\left(\mathrm{E}_{\mathrm{Up} 1}, \mathrm{E}_{\mathrm{Up} 2}\right)$. As shown in Figure 8, more defects are detected in the unpassivated samples for a larger reverse bias of $\mathrm{V}_{\mathrm{R}}=-4$. $\mathrm{V}$.

Native defects are primarily concerned with vacancies, interstitials, and antisite defects. If one assumes that in $\mathrm{GaAs}$, the majority of gallium vacancies $\left(\mathrm{V}_{\mathrm{Ga}}\right)$ recombine with gallium interstitials $\left(\mathrm{Ga}_{\mathrm{i}}\right)$ through Frenkel mechanism, therefore only a small number of remaining $\mathrm{V}_{\mathrm{Ga}}$ react with arsenic interstitials $\left(\mathrm{As}_{\mathrm{i}}\right)$ to form $\mathrm{As}_{\mathrm{Ga}}$ antisites, referred to as EL2. ${ }^{24,25}$ The capture mechanism of interstitials $\left(\mathrm{Ga}_{\mathrm{i}}\right.$ and $\mathrm{As}_{\mathrm{i}}$ ) at dislocations has been observed to create arsenic vacancies $\left(\mathrm{V}_{\mathrm{As}}\right)$ and gallium vacancies $\left(\mathrm{V}_{\mathrm{Ga}}\right){ }^{25-27}$ The LDLTS results indicate that $\mathrm{E}_{\mathrm{UP} 5}$ for $\mathrm{V}_{\mathrm{R}}=-0.25$ and $\mathrm{E}_{\mathrm{UP}}$ for $\mathrm{V}_{\mathrm{R}}=-4 \mathrm{~V}$ have the same origin. The associated peak is well identified in the literature ${ }^{24}$ with its known energy and capture cross-section. In unpassivated samples, it could originate from the GaAs layers and can thus be assigned to the well-known electron trap EL2 in GaAs. ${ }^{24}$

The incorporation of $\mathrm{Te}$ at the interface between $\mathrm{GaSb}$ and GaAs could favour the formation of complexes involving vacancies, antisites, and interstitials. According to the literature, ${ }^{28}$ the self-diffusion in GaSb occurs through the following reaction:

$$
\mathrm{Ga}_{\mathrm{Ga}} \mathrm{V}_{\mathrm{Sb}} \rightarrow \mathrm{V}_{\mathrm{Ga}} \mathrm{Ga}_{\mathrm{Sb}},
$$

where $V_{\mathrm{Sb}}$ being unstable, the pair $\mathrm{Ga}_{\mathrm{Ga}} \mathrm{V}_{\mathrm{Sb}}$ converts into $V_{\mathrm{Ga}} \mathrm{Ga}_{\mathrm{Sb}}$ in which $\mathrm{Ga}$ forms an antisite leaving a gallium vacancy behind. When Te is incorporated into GaSb, it occupies a $\mathrm{V}_{\mathrm{Sb}}$ site, forming the complex $V_{G a} T e_{S b}$ giving to Te the acceptor character.

If $T e$ acts as a donor then the following reaction will result: ${ }^{25}$

$$
\mathrm{Te}+\mathrm{V}_{\mathrm{As}} \rightarrow \mathrm{Te}_{\mathrm{As}}^{+}+\mathrm{e}^{-}
$$

According to this assumption, in the passivated samples Te atoms could create a channel of free electrons. The low $V_{\text {on }}$ in compensated samples observed in this work and in Ref. 12 can therefore be explained by this model involving the presence of $T e$ atoms. Te could also react with $V_{G a}$ to form $T e_{A s} V_{G a}$ complex, which act as an acceptor. These complexes which have been detected by using forward bias voltage conditions are not reported here but will be discussed in a future paper. It is worth noting that this acceptor level has not been detected in unpassivated samples which do not contain $\mathrm{Te}$. One can conclude from this analysis that the incorporation of $T e$ creates additional states in the passivated samples. This is further confirmed from $\mathrm{C}-\mathrm{V}$ and $\mathrm{C}-\mathrm{f}$ analysis.

The DLTS spectra obtained on passivated samples are displayed in Figure 9. Clearly, new peaks appear as displayed in the inset of Figure 9. These additional peaks, which were absent in the unpassivated samples, start appearing for a reverse bias of $V_{R} \geq-0.25 \mathrm{~V}$. Figure 10 shows the Arrhenius plots of six peaks obtained by Laplace DLTS in the passivated samples for a $V_{R}=-0.25 \mathrm{~V}$ (region probed is close to the interface). These six new defects $\left(\mathrm{E}_{\mathrm{p} 1}, \mathrm{E}_{\mathrm{p} 2}, \mathrm{E}_{\mathrm{p} 3}\right.$, $\mathrm{E}_{\mathrm{p} 4}, \mathrm{E}_{\mathrm{p} 5}$, and $\mathrm{E}_{\mathrm{p} 6}$ ) are directly or indirectly related to $T e$ 


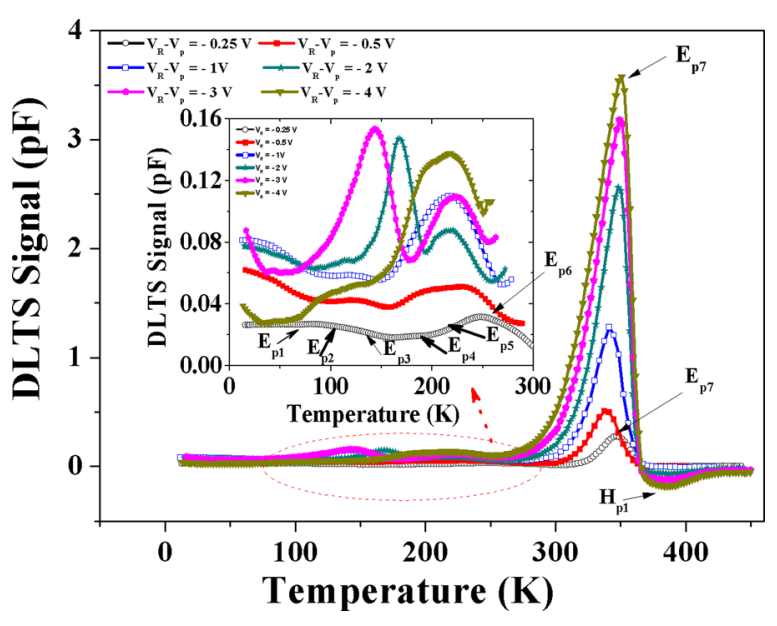

FIG. 9. DLTS measurements of passivated samples at different reverse biases from $\mathrm{V}_{\mathrm{R}}=-0.25 \mathrm{~V}$ to $\mathrm{V}_{\mathrm{R}}=-4 \mathrm{~V}$. The filling pulse characteristics were: $V_{p}=0 \mathrm{~V}, t_{p}=1 \mathrm{msec}$, and rate window $=200 \mathrm{~s}-1$. For clarity, the low temperature peaks are shown in the inset.

doping since these were not observed in unpassivated samples. The most striking feature in Figure 9 is the shape of the peaks in passivated samples (temperature $350 \mathrm{~K}-420 \mathrm{~K}$ ) as compared to those obtained in the unpassivated samples. However, while the peak extends on both sides in the unpassivated devices, when the reverse bias is increased in the passivated structure, the peaks enlarges mainly on the left hand side, whereas an increasing contribution from hole capture-emission shows up on the right hand side as a negative peak.

It can also be noted from Figure 9 that at a reverse bias of $\mathrm{V}_{\mathrm{R}}=-4 \mathrm{~V}$, electron and hole peaks are prominent in the temperature range of $350-420 \mathrm{~K}$, particularly under a large reverse bias. While the hole peak is not detected at small reverse bias of $\mathrm{V}_{\mathrm{R}}=-0.25 \mathrm{~V}$ in passivated samples.

The Arrhenius plot for the reverse bias of $V_{R}=-4 \mathrm{~V}$ is shown in Figure 11. It can be seen that the electron peak in the passivated sample at reverse bias of $-4 \mathrm{~V}$ (Arrhenius peak $E_{p 7}$ shown in Figure 11) is the same as the one recorded at the small reverse bias $\mathrm{V}_{\mathrm{R}}=-0.25 \mathrm{~V}$ (Arrhenius peak $\mathrm{E}_{\mathrm{p} 7}$ from Figure 10), and has the same origin (EL2) as it was already discussed in the case of the unpassivated sample. On

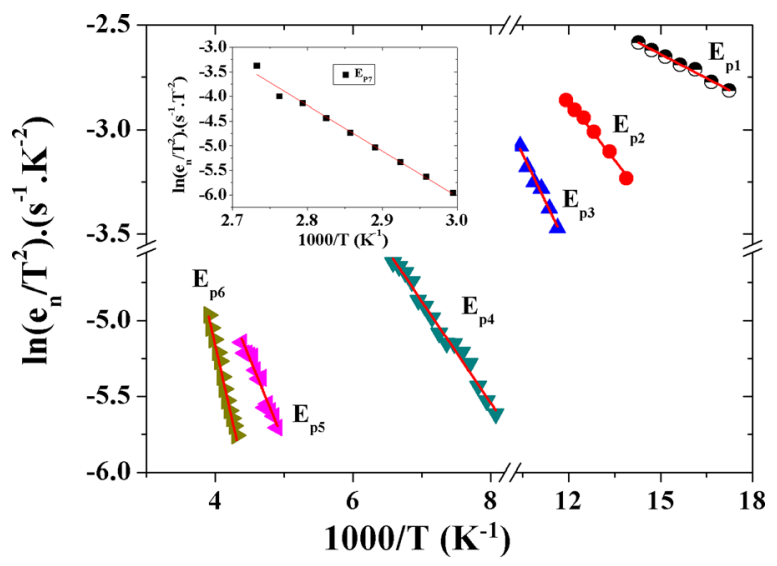

FIG. 10. Arrhenius plots obtained from Laplace DLTS spectra of passivated samples at reverse bias $\mathrm{V}_{\mathrm{R}}=-0.25 \mathrm{~V}$. The inset shows Arrhenius plot of DLTS peak at $350 \mathrm{~K}$. the other hand, the hole peak $\left(\mathrm{H}_{\mathrm{p} 1}\right.$ shown in Figure 11) with an activation energy of $\sim 0.72 \mathrm{eV}$ was previously reported in undoped $\mathrm{GaSb}^{29}$ It is attributed to the interdiffusion of Te in undoped $\mathrm{GaSb}$; in our case, Te is present at the interface of $\mathrm{GaSb}$ and $\mathrm{GaAs}$ in passivated samples and might diffuse to GaSb side.

The activation energies, capture cross-section, and defects concentrations are summarised in Table I for a reverse bias $\mathrm{V}_{\mathrm{R}}=-0.25 \mathrm{~V}$ that allows probing the region close to the interface between GaAs and GaSb. As shown from both Figures 6 and 9 increasing $V_{R}-V_{p}$ leads to a very unique behaviour that is not possible to attribute to a single level. In other words, we can no longer invoke the EL2 as the observed signal shifts to higher temperatures and distorts. Laplace DLTS shown in Figures 8 and 11 detect more than one level in both samples when we increase $V_{R}-V_{p}$. Such a behavior is very likely to be due to interface states. This can easily be explained by the following: increasing the voltage pulse height will increase the energy range from which the carriers emit to the conduction band on one side (GaAs) and to the valence band on the other side ( $\mathrm{GaSb}$ ). These emission rates are energy-dependent. The situation becomes also complex as we must bear in mind that for interface states, the capture cross-section is not only temperature dependent but also energy dependent. This is the reason for distortion. In other words, some states have larger capture cross sections in a given energy range than elsewhere. These various contributions increase the capacitance transient by increasing the components of the various transients and shift the peak position due to the fact that the overall transient is far from the ideal exponential that should be observed for a single trap, no matter how large is the reverse bias. As a consequence, a small voltage pulse $V_{R}-V_{p}$ would be preferred for minimizing the shift of the peak and increasing the energy resolution.

In order to correlate the relationship between $C V$ and DLTS of both passivated and unpassivated samples, one considers the areas below the DLTS signals recorded under the same conditions. This allows the determination of their ratio, which matches surprisingly with the ratio of the intercepts in Figure 4. Therefore, both the intercepts obtained from the $C V$ analysis and the deep DLTS peaks are very likely due to

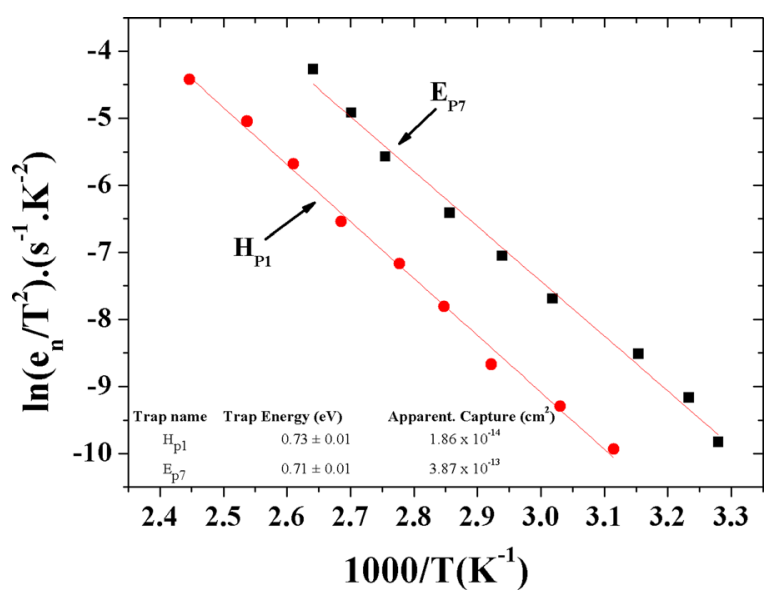

FIG. 11. Arrhenius plots derived from conventional DLTS for electron and hole traps detected in passivated samples at a reverse bias $\mathrm{V}_{\mathrm{R}}=-4 \mathrm{~V}$. 
TABLE I. Summary of trap parameters for both unpassivated and Te-Passivated samples at $V_{R}=-0.25 V, V_{P}=0 \mathrm{~V}$, and $t_{p}=1 \mathrm{msec}$ and $V_{R}=-4 V$, $\mathrm{V}_{\mathrm{P}}=0 \mathrm{~V}$, and $\mathrm{t}_{\mathrm{p}}=1 \mathrm{msec}$.

\begin{tabular}{|c|c|c|c|c|}
\hline Sample name with experimental conditions & Trap name & $\mathrm{E}_{\mathrm{T}}(\mathrm{eV})$ & Capture cross-section, $\sigma\left(\mathrm{cm}^{2}\right)$ & Trap concentration $\left(\mathrm{cm}^{-3}\right)$ \\
\hline $\mathrm{V}_{\mathrm{R}}=-0.25 \mathrm{~V}, \mathrm{~V}_{\mathrm{P}}=0 \mathrm{~V}$ Unpassivated & $\mathrm{E}_{\mathrm{Up} 5}$ & $0.79 \pm 0.005$ & $1.14 \times 10^{-12}$ & $3.09 \times 10^{14}$ \\
\hline \multirow[t]{5}{*}{$\mathrm{V}_{\mathrm{R}}=-4 \mathrm{~V}, \mathrm{~V}_{\mathrm{P}}=0 \mathrm{~V}$ Unpassivated } & $\mathrm{E}_{\mathrm{Up} 1}$ & $0.110 \pm 0.005$ & $3.76 \times 10^{-20}$ & $6.10 \times 10^{14}$ \\
\hline & $\mathrm{E}_{\mathrm{Up} 2}$ & $0.130 \pm 0.001$ & $1.29 \times 10^{-19}$ & $7.38 \times 10^{14}$ \\
\hline & $\mathrm{E}_{\mathrm{Up} 3}$ & $0.164 \pm 0.005$ & $2.52 \times 10^{-20}$ & $7.56 \times 10^{14}$ \\
\hline & $\mathrm{E}_{\mathrm{Up} 4}$ & $0.402 \pm 0.005$ & $1.90 \times 10^{-17}$ & $1.29 \times 10^{15}$ \\
\hline & $\mathrm{E}_{\mathrm{Up} 5}$ & $0.78 \pm 0.01$ & $2.12 \times 10^{-13}$ & $5.63 \times 10^{15}$ \\
\hline \multirow[t]{7}{*}{$\mathrm{V}_{\mathrm{R}}=-0.25 \mathrm{~V}, \mathrm{~V}_{\mathrm{P}}=0 \mathrm{~V}$ Te-passivated } & $\mathrm{E}_{\mathrm{p} 1}$ & $0.007 \pm 0.001$ & $2.05 \times 10^{-21}$ & $2.53 \times 10^{13}$ \\
\hline & $\mathrm{E}_{\mathrm{p} 2}$ & $0.016 \pm 0.001$ & $4.89 \times 10^{-21}$ & $2.52 \times 10^{13}$ \\
\hline & $\mathrm{E}_{\mathrm{p} 3}$ & $0.026 \pm 0.002$ & $9.89 \times 10^{-21}$ & $2.50 \times 10^{13}$ \\
\hline & $\mathrm{E}_{\mathrm{p} 4}$ & $0.058 \pm 0.004$ & $7.61 \times 10^{-21}$ & $2.28 \times 10^{13}$ \\
\hline & $\mathrm{E}_{\mathrm{p} 5}$ & $0.096 \pm 0.004$ & $7.15 \times 10^{-21}$ & $2.53 \times 10^{13}$ \\
\hline & $\mathrm{E}_{\mathrm{p} 6}$ & $0.173 \pm 0.002$ & $1.58 \times 10^{-19}$ & $2.93 \times 10^{13}$ \\
\hline & $\mathrm{E}_{\mathrm{p} 7}$ & $0.75 \pm 0.004$ & $4.81 \times 10^{-12}$ & $2.51 \times 10^{14}$ \\
\hline \multirow[t]{2}{*}{$\mathrm{V}_{\mathrm{R}}=-4 \mathrm{~V}, \mathrm{~V}_{\mathrm{P}}=0 \mathrm{~V}$ Te-passivated } & $\mathrm{E}_{\mathrm{p} 7}$ & $0.71 \pm 0.014$ & $3.87 \times 10^{-13}$ & $3.17 \times 10^{15}$ \\
\hline & $\mathrm{H}_{\mathrm{p} 1}$ & $0.73 \pm 0.011$ & $1.86 \times 10^{-14}$ & $1.22 \times 10^{14}$ \\
\hline
\end{tabular}

the interface states induced by the remaining dislocations generated by the strain energy that is not fully relieved by the $90^{\circ} \mathrm{IMF}$ dislocations to which we must add the proper role of $T e$ atoms incorporated to compensate those dislocations. However, further $C V$ at different frequencies/temperatures and DLTS with varying $V_{R}$ and $V_{P}$ experiments are planned to elucidate further the role of interfacial states. These results will be discussed in a future paper.

\section{CONCLUSION}

In summary, Current-Voltage characteristics in the reverse bias condition show that the leakage current is higher in passivated samples than in unpassivated ones for values of voltages greater than $-1.5 \mathrm{~V}$. Moreover, a lower $V_{\text {on }}$ in the passivated samples could be attributed to more defects than in the unpassivated samples. The $C V$ analysis and C-f measurements also confirms that the incorporation of $T e$ at the interface results in an increase of the interface states. This is also supported by the larger number of traps detected by DLTS in the passivated samples near the interface between GaAs and GaSb. Among these traps, EL2 is the main midgap defect detected in both unpassivated and passivated samples. This EL2 trap plays a role near the interface between GaAs and GaSb. Other studies on similar samples have shown that Te plays a role in improving the structural properties of the interface by reducing the dislocation density. However, it is clear from this work that the electrical properties of the passivated samples are not as good as those of the unpassivated devices in terms of the number of electrically active defects.

${ }^{1}$ M. Li, Y. Qiu, G. Liu, Y. Wang, B. Zhang, and L. Zhao, J. Appl. Phys. 105, 094903 (2009).

${ }^{2}$ G. Delhaye, L. Desplanque, and X. Wallart, J. Appl. Phys. 104, 066105 (2008).

${ }^{3}$ R. B. Laghumavarapu, A. Moscho, A. Khoshakhlagh, M. El-Emawy, L. F. Lester, and D. L. Huffaker, Appl. Phys. Lett. 90, 173125 (2007).
${ }^{4}$ M. Geller, C. Kapteyn, L. Müller-Kirsch, R. Heitz, and D. Bimberg, Phys. Status Solidi B 238, 258 (2003).

${ }^{5}$ P. S. Dutta, H. L. Bhat, and V. Kumar, J. Appl. Phys. 81, 5821 (1997).

${ }^{6}$ S. H. Huang, G. Balakrishnan, A. Khoshakhlagh, A. Jallipalli, L. R. Dawson, and D. L. Huffaker, Appl. Phys. Lett. 88, 131911 (2006).

${ }^{7}$ W. Qian, M. Skowronski, R. Kaspi, M. De Graef, and V. P. Dravid, J. Appl. Phys. 81, 7268 (1997).

${ }^{8}$ A. Y. Babkevich, R. A. Cowley, N. J. Mason, and A. Stunault, J. Phys.: Condens. Matter 12, 4747 (2000).

${ }^{9}$ A. Jallipalli, G. Balakrishnan, S. H. Huang, A. Khoshakhlagh, L. R. Dawson, and D. L. Huffaker, J. Cryst. Growth 303, 449 (2007).

${ }^{10}$ L. Wang, M. Li, M. Xiong, and L. Zhao, Nanoscale Res. Lett. 4, 689 (2009).

${ }^{11}$ A. Jallipalli, K. Nunna, M. N. Kutty, G. Balakrishnan, G. B. Lush, L. R. Dawson, and D. L. Huffaker, Appl. Phys. Lett. 95, 072109 (2009).

${ }^{12}$ A. Jallipalli, K. Nunna, M. N. Kutty, G. Balakrishnan, L. R. Dawson, and D. L. Huffaker, Appl. Phys. Lett. 95, 202107 (2009).

${ }^{13}$ P. Chattopadhyay and D. P. Haldar, Appl. Surf. Sci. 143, 287 (1999).

${ }^{14}$ Physics of Semiconductor Devices, 3rd ed., edited by S. M. Sze and K. K. $\mathrm{Ng}$ (John Wiley \& Sons, Inc., 2007).

${ }^{15}$ Heterojunction Band Discontinuities, Physics and Device Applications, edited by F. Capasso and G. Margaritondo (North-Holland, 1987).

${ }^{16}$ S. Pandey and S. Kal, Solid State Electron. 42, 943 (1998).

${ }^{17}$ J. Bardeen, Phys. Rev. 71, 717 (1947).

${ }^{18}$ R. Padma, B. P. Lakshmi, and V. R. Reddy, Superlattices Microstruct. 60, 358 (2013).

${ }^{19}$ A. Sellai and M. Mamor, Phys. Status Solidi C 8, 1611 (2011).

${ }^{20}$ D. Lang, J. Appl. Phys. 45, 3023 (1974).

${ }^{21}$ L. Dobaczewski, P. Kaczor, I. D. Hawkins, and A. R. Peaker, J. Appl. Phys. 76, 194 (1994).

${ }^{22}$ R. J. Kaplar, S. A. Ringel, S. R. Kurtz, J. F. Klem, and A. A. Allerman, Appl. Phys. Lett. 80, 4777 (2002).

${ }^{23}$ D. Pons and S. Makram-Ebeid, J. Phys. France 40, 1161 (1979).

${ }^{24}$ A. C. Irvine and D. W. Palmer, Phys. Rev. Lett. 68, 2168 (1992).

${ }^{25}$ D. T. J. Hurle, J. Cryst. Growth 237, 1621 (2002).

${ }^{26}$ C. Frigeri, J. L. Weyher, and J. Jimenez, J. Phys.: Condens. Matter 12, 10335 (2000).

${ }^{27}$ E. R. Weber, H. Ennen, U. Kaufmann, J. Windscheif, and J. Schneider, J. Appl. Phys. 53, 6140 (1982).

${ }^{28}$ L. Hui, Z. Kai, P. Jingbiao, S. Yundong, W. Zhu, and Z. Youwen, Semicond. Sci. Technol. 26, 075016 (2011).

${ }^{29}$ E. Kuramochi, N. Kondo, Y. Takanashi, and M. Fujimoto, Appl. Phys. Lett. 63, 2664 (1993). 